\title{
Agency, Scarcity, and Mortality
}

\author{
Luca Ferrero \\ Philosophy \\ UW-Milwaukee \\ PO Box 4I 3 \\ Milwaukee, WI 5320I-64I3 \\ luca.ferrero@gmail.com \\ http://people.uwm.edu/ferrero
}

November 27, 2015

\author{
Version I.04 - forthcoming in Journal of Ethics \\ (C) 2015 Luca Ferrero
}




\title{
Agency, Scarcity, and Mortality
}

\begin{abstract}
It is often argued, most recently by Samuel Scheffler, that we should reconcile with our mortality as constitutive of our existence: as essential to its temporal structure, to the nature of deliberation, and to our basic motivations and values. Against this reconciliatory strategy, I argue that there is a kind of immortal existence that is coherently conceivable and potentially desirable. First, I argue against the claim that our existence has a temporal structure with a trajectory that necessarily culminates in an ending. This claim is based on two false assumptions: that a life as a whole calls for narrative structure, and that narratives necessarily require closure as temporal endings. Second, I reject the proposal that temporal finitude is constitutive of the basic elements of diachronic agency, including the nature of deliberation and of our values. I argue that only finitude as scarcity of opportunities is constitutive of these elements. Additionally, scarcity might be present in an endless existence. Therefore, it is not incoherent to conceive of a recognizable and potentially desirable immortality that grounds the core features of diachronic agency. Thus, against the reconciliatory strategy, I conclude that we might never fully reconcile with mortality. Although we might embrace our inescapable mortality as essential to a fuller range of features of our existence, we can still justifiably regret our missing on an immortal existence.
\end{abstract}

\section{Introduction}

\section{I Reconciling with Our Mortality}

I.I.I When we contemplate the prospect of our death, it is not uncommon to respond with fear, despair, or angst. Even if we do not go as far as Tolstoy (I 884) in thinking that a finite existence is ultimately futile and meaningless, we might still lament

I For conversations, comments, and criticisms, I would like to thank Clotilde Calabi, John Martin Fisher, Jay L. Garfield, Peter J. Graham, Masaki Ichinose, Joshua Landy, Benjamin Mitchell-Yellin, Richard Moran, Elisa Paganini, Eric Schwitzgebel, Alessandro Zucchi, and audiences at the University of Milan, the University of Tokyo, and the Immortality Project capstone conference at UC-Riverside. A special thank to John Martin Fischer and his team for their wonderful job in directing and organizing the 'Science and Philosophy of Immortality' project. This paper was written with the generous support of the John Templeton Foundation. The views presented in this paper do not necessarily reflect the views of the John Templeton Foundation. 
and mourn the necessary finitude of our life and long for an endless existence.

Further reflection on the nature of our life might, however, convince us otherwise. Our mortality might turn out to be essential to our kind of existence: temporal finitude might be a central constitutive factor of our cares and values, of our basic motivation to act, and of the meaningfulness of our life. ${ }^{2}$

Ultimately, the very idea that we might have an endless life, or at least a value-laden one, might be conceptually incoherent. If so, we should reconcile ourselves with our mortality. We should no longer mourn our finitude, and we should stop desiring something-an immortal existence-that is a "fantasy ... inherently confused and in principle unsatisfiable," (Scheffler 20I3: 208).

I. I.2 The most articulate and promising defense of this 'reconciliatory strategy,' as I will call it, has been recently offered by Scheffler (2OI3). Scheffler argues that temporal finitude is constitutive of such things as the characteristic temporal structure of our existence, the fundamental structure of deliberation and its objects, the basic motivation to engage in any activity whatsoever, the development of a sense of self, and the nature of many of our cares, values, and virtues. In Scheffler's words: “Our lives are so pervasively shaped by the understanding of them as temporally limited that to suspend that understanding would call into question the conditions under which we value our lives and long for their extension." From which he concludes, "we need to die... because an eternal life would, in a sense, be no life at all," Scheffler (2013: $95-6)$.

I.I.3 In this paper, I will argue that these attempts at reconciliation are not fully satisfactory. Although they correctly call at-

${ }^{2}$ See Malpas (1 998), May (2009), Nussbaum (1 989), Nussbaum (I 994: 225-32)-but compare later recantation in Nussbaum (1999) and Nussbaum (2OI3)-Scheffler (2OI3), and Smuts (2OII). This suggestion is also part of the famous discussion of immortality in Williams (1973), although many of the responses to Williams focus on his claim about the undesirability of immortality as boring rather than on claims about its inconceivability or unrecognizability. 
tention to the core features of our temporal existence, they fail to prove that mortality-as the necessary temporal finitude of our existence-is truly constitutive of them. I will argue that there is another dimension under which we are primarily finite and bounded-the scarcity of the opportunities for action. This scarcity is the necessary and often sufficient condition for the conceivability and recognizability of a temporally extended existence. If so, there is a kind of immortality that we might be able to coherently conceive and possibly desire. Even if we acknowledge that many valuable aspects of our existence are intimately related to our mortality and that our death is inescapable, we might still be able to make sense of our longing for immortality. If so, we might never fully reconcile with the prospect of our eventual death. Or so I will argue.

I.I.4 I will frame my discussion around the two main themes in Scheffler's defense of the reconciliatory strategy. First, I will consider whether our existence has a temporal structure with a characteristic trajectory that necessarily culminates in an ending. Special attention will be given to the suggestion that our lives necessarily call for a narrative structure and that this structure can achieve closure only by our dying. I will argue that the narrative proposal has some merit in highlighting the distinctive temporal unity of portions of our existence, but it is mistaken in claiming that this unity should embrace a whole life. Additionally, the narrative proposal is mistaken in thinking that narrative closure should necessarily take the form of a temporal ending. There can be closure in an activity or in a portion of one's life in the form of a 'dynamic resolution'-that is, the proper fitting of the temporal elements in a cross-temporal pattern, a fitting that is still compatible with (and at times might actually promote) the continuation of the activity or existence in question.

Second, I will consider the proposal that temporal finitude is a necessary constitutive factor of the basic elements of our diachronic agency, including the distinctive character of our practical predicament, the nature and structure of deliberation, and the basic cares and values that guide our conduct. I will argue that the truly necessary factor is the scarcity of opportunities for action and that this scarcity might in principle be present even in an endless existence. Therefore, I will claim, it is not conceptually 
incoherent to think of an immortal existence that still grounds the core aspects of our existence as diachronic agents. Against the reconciliatory strategy, I contend that there is at least one conceivable (and potentially desirable) kind of immortal existence and thus we cannot be fully reconciled with our mortality as the necessary precondition of the coherent conceivability of any recognizable existence.

I will conclude by claiming that, even if we might embrace our inescapable mortality as essential to a fuller range of features of our human existence, in our capacity as diachronic agents, we might still harbor a justifiable regret for our missing on a particular kind of immortal existence.

I. I.5 My conclusion rests on a conjecture about the conceivability of a particular kind of immortal life. It is notoriously difficult to offer a conclusive proof about conceivability claims, even more so for claims that rest on such notions as agency, personal identity, and the idea of an infinite amount of time. However, I hope that my considerations are at least successful in shifting the burden of proof back onto the reconciliatory strategy.

In order to be fully reconciled with our mortality, therefore, we still need to be shown that there could be no room for any coherent longing for some kind of conceivable endless existence. But lacking a convincing argument to that effect, we are justify in remaining ambiguous about the prospect of our inevitable demise. Although much of what we find choiceworthy and valuable in our finite lives might necessarily depend on our mortality, I will argue that an immortal life under scarcity of opportunities still exhibits the distinctive and desirable basic features of our diachronic agency. Being deprived of this kind of immortal life, therefore, is something that we might still find regrettable and deplorable, even while embracing many of the things that only our mortality could offer us.

I. I.6 Before proceeding, a couple of clarifications are in order. In this paper, by 'mortality' I mean the 'necessary temporal finitude' of our individual existence-that is, the fact that our lives have not only a beginning but also an inevitable temporal upper bound. What is under discussion is not any specific finite length that our 
lives might have, but the general fact that they are necessarily temporally finite and thus, sooner or later, they terminate for good.

When I talk about 'immortality' I am only referring to an indefinitely long individual or personal existence. I am not going to discuss what Scheffler (2O13) calls the 'collective afterlife'the possibility that, although individual lives are necessarily finite, humanity will continue to exist forever. In addition, in order to simplify the discussion and focus on the question of the basic relationship between finitude and the structure of agency, I will make a few assumptions. Whenever I talk about immortal existence I assume that all subjects are immortal and that: they all know to be immortal, they do not age, and they do not necessarily face an 'over-population' problem. Finally, I am interested in lives that extend in time. Hence, when talking about immortality I have in mind 'sempiternal' or 'everlasting' existence rather than an atemporal or timelessly 'eternal' one. ${ }^{3}$

\section{Mortality and Narrative}

\section{I Narrative and Temporal Unity}

2.I.I Scheffler (2013: 96) correctly remarks that, as biological organisms, our existence is characterized by a basic division in distinct stages, including those of "birth, maturation, deterioration, and death," each of which comes with its "characteristic tasks, challenges, and potential rewards."

Could this observation help us reconcile with our mortality? It does not seem so. That our biological development frames many of our substantive concerns does not show that we could not conceive and desire an existence free of these biological imperatives, especially those of eventual deterioration and death. It seems

3 Those writing on immortality are not always careful about this distinction and use 'eternal life' to refer that a life with an infinite duration in time, that is, to what should be more properly called a 'sempiternal life.' This is the case, for instance, of the quote from Scheffler (201 3: 95) above. For more on the distinction between eternity and sempiternity, see Helm (2014). 
plausible that we could conceive and desire an existence that is perpetually stuck at the mature stage. Biological deterioration and eventual death appear to be unfortunate and regrettable features of our existence. And the fact that they are inevitable provides little, if any, consolation for our mortality.

2.I.2 When Scheffler (2013: IOO) recapitulates his view on the necessary stages of human life, however, he no longer presents the stages in biological terms. He talks about a more general temporal structure: "it is essential to our idea of a life that it is temporally bounded, with a beginning, a middle, and an end." Although it might not accord with Scheffler's intention, this passage could be interpreted along the lines of the 'narrative view' of mortality. According to this view-championed among others by Nussbaum (1994), Malpas (1998), May (2009), and Ismael (2006)-our lives are structured by narratives, and an endless existence could never reach the closure to which our mortal lives aspire on account of their narrative structures. ${ }^{4}$

There is no guarantee that death gives us closure, of course. Death might be untimely, occurring either too soon (prior to our reaching the proper ending of our life-narrative) or too late (after our existence has already reached its narrative closure and it is just dragging on). But we should welcome our mortality as the necessary precondition for the possibility of reaching a timely closure in the master narrative of our existence. Or so the narrative view claims.

4 The connection between human mortality and narrative closure plays a prominent role in the classic discussions of narrative by Kermode (2000) and Brooks (2OI 2). For them, a narrative with closure as a temporal ending helps us gain an understanding of the distinctive temporality of our existence and to confront its necessary finitude. A narrative-cumclosure is our distinctive way of coping with and trying to make sense of our mortality, once we realize that it is our inescapable condition. It is important to notice that their order of explanation is the reverse of the narrative view I am discussing. According to the narrative view, given that our lives could only make sense in terms of a narrative-cum-closure, we should come to accept the temporal boundedness of our existence as the only temporal structure that would give us narrative closure and the understanding associated with it. For Kermode and Brooks, instead, given that we are necessarily mortal, we should help ourselves of the resources of narrative to deal with mortality. 
2.I.3 Unfortunately, there are two basic problems with this account. First, it is highly controversial whether lives require a master narrative that covers their entire span. ${ }^{5}$ Even if narratives might be widespread in guiding, explaining, and describing many activities in which we engage, guidance by a single master narrative is not necessary in order to have a recognizable and desirable life. This conclusion does not rule out that some agents might have or seek a master narrative that covers their entire lives, and that they might, thereby, find some comfort in their mortality. But this is hardly a recipe for all.

2. I.4 Second, even if it were true that our kind of temporally extended existence requires a master narrative, it does not follow that narrative closure can only come to a finite life. Contrary to what the narrative view claims, a narrative does not require a closure in the form of a terminus - a temporal end point. As I have argued in Ferrero (2009), narrative closure can be attained in the form of a dynamic and ongoing resolution, a resolution that is compatible with the continuation of an activity and that might actually propel it further (for instance, take the case of a potentially endless series of harmonic resolutions in a musical composition like a perpetuum mobile). A narrative achieves closure when its elements come to fit together in a pattern that extends over time and that confers a distinctive significance to these elements. But the completion of the pattern-the closure that is reached when all the elements are properly fitting into place-does not have to bring the narrative, and what the narrative describes and explains, to a temporal stop.

Hence, even if a recognizable and desirable human life would have to take a narrative form, this would not help us with mortality. The presence of a narrative might move us to make sure that we reach its closure, but this closure might take the dynamic form that allows for both the narrative and the existence to continue into an indefinite future, while continuing to seek a series of dynamic closures that might continue to propel both the narrative and the existence forward ad infinitum. As a result of these closures, the narrative might go through changes and transformations, but it might never demand that it be eventually completed

\footnotetext{
s See for instance Strawson (2004) and Williams (2009).
} 
and terminated once and for all. ${ }^{6}$

2.I.5 Notice that the trouble with the narrative view concerns only the application of a single master narrative to an entire life. I am not objecting to the central role that narratives play within lives. As I argued in Ferrero (2009), narratives provide the basic forms of description and explanation of temporally extended intentional activities. This is because narratives are distinctively apt at conveying the basic features of temporally unified activities. Let me explain.

An activity can extend over time by mere continuity. In this case, each momentary action sets the stage for the following momentary action, by creating or preserving the conditions under which the following action takes place. A momentary action might also indirectly affect more distant actions by delayed causal effects. But throughout this series of actions, there is no attempt by the agent at securing any integration of the activity as a temporal whole that extends over an interval that embraces both past and future times. The activity simply flows from moment to moment, without any regard for its overall shape.

By contrast, a 'temporally unified activity' is one in which the various stages are undertaken over an extended interval in light of their expected contribution to cross-temporal patterns of actions, experiences, and attitudes; patterns that give significance to these very actions, experiences, and attitudes-first of all as the actions, experiences, and attitudes of the selfsame subject. A temporally unified activity is one whose different momentary stages relate to each other in a coherent and cohesive way that makes possible the realization of the cross-temporal patterns. A perspicuous description of such an activity needs to show how its various stages fit together in this distinctive way.

The contribution of the elements can be appreciated only when they are seen as parts of the overall cross-temporal pattern that they bring about, that is, as part of the performance of the temporally unified activity itself. A narrative is specifically suited to describe and explain a temporally unified activity as temporally

\footnotetext{
${ }^{6}$ For further criticisms of the narrative view of mortality, see Fischer (2005) and Rosati (2013: \$3.3).
} 
unified. This is because a narrative description uses devices that highlight not just the causal connections between the described events but also the cross-temporal patterns in which they enter, which helps us see their teleological contribution to the success of the activity and their significance both within the activity itself and, more generally, within the agent's larger plans (contrast a narrative with the description provided by a simpler chronicle, which only describes the stages in their temporal sequence with no indication of their mutual connections but for their relative standing in the temporal sequence).

\subsection{Temporal Horizons of Integration}

2.2. I The distinction between mere continuity and temporal unity applies not just to activities but also to portions of the agent's life (if not to the entire life itself). In mere continuity, the momentary stages of the agent's existence follow each other without any attempt at cross-temporal integration. In a temporally unified stretch of existence, instead, the various stages are supposed to relate to each other over that temporal interval so as to secure some diachronic integration. ${ }^{7}$

Let's call the stretch of one's own existence over which an agent is subjected to pressures for diachronic integration the 'interval of temporal identification and integration.' These pressures take various forms: demands to live up to the intentions one has acquired and the commitments one has undertaken; pressures to take responsibility and being held accountable for one's past actions; demands to agglomerate and integrate one's pursuits to avoid conflicts (which might make their joint pursuit either impossible or too costly).

There might be different kinds of temporal integration, with various degrees of tolerance of conflicts and inconsistencies, but

${ }^{7}$ I find much to agree with the characterization of the structure of unity offered in Malpas (1998), including the connection with narrative, but Malpas is mistaken in thinking that this structure applies to a life as a whole and thus that a life as a whole is to be organized around a narrative. This mistake, in turn, invalidates much what he says about the necessary role played by mortality in giving significance to our lives. 
for present purposes we do not need to discuss any particular standard or model of diachronic integration. Our concern is only with the general outline of the demands of temporal unity as opposed to those of mere continuity.

2.2.2 The horizon of temporal identification and integration need not extend to the agent's entire life. There might be distal portions of one's existence to which one presently relates only by mere continuity. Although the continuity might be sufficient for temporal identity, the lack of the stronger kind of temporal identification and integration limits the pressures that come from and are directed toward the portions that fall outside the horizon of temporal identification. For instance, consider the 'outgrowing' of commitments as one gets older. Leaving behind certain projects as we mature is a typical instance of the changes in the temporal horizon of our identification and integration. Even if we do not lose the direct and first-person psychological access to our younger selves, that access no longer carries the pressures (both motivational and rational) toward temporal integration that we still acknowledge from a more recent past.

A similar distinction might also apply to projections and anticipations concerning our future selves. Very distant selves might be taken to be outside of the reach of one's present commitments, projects, cares, and values. One might anticipate that, at some point, along the line of temporal continuity, one will have gradually come to outgrow, move away, or simply lose one's present commitments and, more generally, one's present practical standpoint. When so, there is a limit to the temporal reach, both past and future, of one's present demands for temporal integration. This is not an unfamiliar scenario within the standard temporal span of our lives. But it is likely to become a standard feature of lives that last much longer than our ordinary ones, and a fortiori of immortal lives.

2.2.3 How does the temporal integration within these intervals relate to the idea of narrative? And how does it bear on our stance toward mortality? Let's begin with narrative.

A master narrative can provide the organizing principle within an interval of temporal integration. When so, there is a substan- 
tive goal or set of goals that exerts overarching guidance over that interval. But temporal integration does not require a single substantive master project. It can just take the form of a more general structural demand: to secure consistency between the various substantive pursuits that one undertakes within that interval against the background of a (relatively) stable practical standpoint. The only narrative that covers the entire interval is the story of 'trying to keep oneself together over that interval as an integrated agent.' This is indeed something one is trying to live up to but only as a structural constraint that frames the more specific and substantive first-order endeavors in which one is engaged.

Notice that the narrative devices of description and explanation still apply to the pursuit of this structural demand. This is because in order to achieve and maintain temporal integration one needs to secure cross-temporal patterns not just within single activities but also across them, in the interest of non-conflictual coordination and agglomeration. The narrative structure of structural integration, however, does not lend support to the idea of closure as temporal termination. Quite the contrary. Temporal integration is a constant work in progress. Whatever closure one might achieve, it is just a temporary matter. Novel challenges are expected to arise because one continues to operate as an agent in time. The diachronic integration that might be achieved at any particular time is not an everlasting achievement. It is rather a paradigmatic example of 'dynamic resolution' (see 4). The closure that one might achieve at any particular time is only the present but momentary success of an ongoing and potentially open-ended process, rather than a place where one can rest on one's laurels.

2.2.4 The trouble with the narrative view of mortality is that it confuses the distinctive structure of ongoing temporal integration that operates throughout our existence, or at least large portions of it, with the temporal unity imposed by a single substantive firstorder narrative with a temporal terminus. Once we get clear on the dynamic character of temporal integration and the associated narrative descriptions and explanations, we are no longer in a position to find consolation for our mortality in the alleged demand that a life be structured around a single master narrative.

2.2.5 Reflection on the structure of ongoing temporal integra- 
tion, however, might suggest a different kind of concern with an endless existence. Could such existence achieve temporal integration across all of its infinite duration? If not, we might be unable to recognize and desire an endless existence.

There seem to be two main problems with the suggestion that temporal integration could embrace a life of indefinite duration. First, could a subject have the psychological resources to keep track and hold together all of the elements of this immortal life? Second, even if the psychological resources were up to this task, could there be cross-temporal patterns of actions and attitudes that extend over the entire span of immortal life?

These questions raise legitimate concerns. As temporally and psychologically finite subjects, we might be unable to project ourselves into an endless existence that tries to achieve temporal integration over its infinite duration. It might be unfathomable to us what it is like to secure integration over that temporal horizon and to have the required psychological resources (see Burley $2009 \mathrm{~b})$. Even so, this does not yet lend support to the idea that temporal finitude is essential to any recognizable existence.

2.2.6 This is because the finitude need not be in the extension of the entire life but only in the temporal horizon of identification and integration. As discussed above, the agent at any particular moment might have a limited temporal interval, both in the past and in the future, over which she is trying to achieve diachronic integration. The temporal limitation might have several compatible sources. For instance, with respect to a certain portion of the past, the agent might have lost access to it, no longer remembering it in a way that would sustain first-person identification. Or she might have outgrown or rejected prior commitments, preferences, cares, and values so that her current practical standpoint no longer puts pressure for identification with this past.

Likewise for the future: there are distal portions of one's future in which one cannot project oneself if not in the form of a most generic anticipation-one expects to be there as a result of mere continuity. The anticipation along mere lines of continuity is still done from a first-person perspective, but only in a 'generic' form, since one might be unable to assign any substantive practical 
standpoint to the distal self that one is projecting into. That is, one might be unable to anticipate which specific commitments, preferences, cares, and values one will have at that future time. When so, one has no substantive grounds on which to try to secure temporal integration from the present time. One is only under a most general and generic prudential pressure to secure the basic preconditions for the success of one's future projects, whatever those might turn out to be.

The temporal limit in the horizon of diachronic identification and integration is a familiar feature in our lives, especially when we consider the relations that we might have to portions of our life several years or decades away from us, both in the past and in the future.

2.2.7 A finite temporal horizon is also a moving one. As the agent travels through time, so does her horizon of integration. Some portions of one's past existence can drop out of it, while some portions of the future existence might get included. The rate at which the horizon changes is not necessarily proportional to the passage of time. But when a substantial amount of time has elapsed, we usually expect a similarly sizable movement and re-centering of the temporal horizon. In addition, the change is not usually limited to the temporal expanse of the reach of the pressures for integration. The change also occurs in the practical standpoint from which the integration is to be pursued, given that the passage of time is likely to alter commitments, preferences, cares, and values.

For a while, the temporal horizons one has at different times might overlap. But over longer temporal intervals, the overlap is likely to cease. When there is no overlap, there are portions of one's past and future that fall outside of the pressure of temporal integration with one's present time and the other portions of one's existence that still fall within the limited temporal horizon of integration. With respect to the portions that fall outside of the horizon, one is no longer under a pressure of integration. One is only under the demands imposed by mere continuity.

2.2.8 The existence of non overlapping horizons of integration generates complicated problems for diachronic practical rationality, which even our best theories of rationality might not be fully 
equipped to deal with. The difficulty is in handling the mixing of the generic prudential demands toward the distal selves, which fall outside of the horizon of integration, together with the more specific demands for temporal integration within the horizon, while knowing that the horizon continues to move and reshape over time.

These complications are likely to be exacerbated in very long lives, especially in indefinitely long ones. ${ }^{8}$ However, whatever difficulties we might encounter in formulating the correct principles of diachronic practical rationality, these difficulties do not show that there is any conceptual incoherence in trying to imagine our projection into longer and potentially endless lives. What might be troubling is to imagine a temporal integration that embraces an infinite life within a single all-encompassing horizon. But what I am suggesting here is that we could imagine a projection into an infinite life as a sequence of partially overlapping, finite, and moving horizons of temporal integration-a projection in which, I contend, we already engage within our own lives when we look at them over long temporal intervals. Even if the temporal horizons of integration are necessarily finite, therefore, this finitude does not tell against the conceivability of an immortal existence.

2.2.9 One might still object to this conceivability on the grounds that over an indefinitely long period of time, an agent is likely to undergo such a massive and radical series of transformations that we are utterly clueless about what she might be like in the long run. We cannot even rely on the evidence of the transformations that we observe in other agents around us. Those are limited to the small range of changes that are possible within the lifespan of humans, which is just a minuscule fraction of an endless existence. ${ }^{9}$

8 Our relations with the distal stages are further complicated by the possibility that, over the long term, we might find our survival to be threatened, even if the changes have been gradual and continuitypreserving. Altshuler (2016) interestingly argues that the existence of this threat might depend on whether we take a forward-looking or a backward-looking perspective toward the distal stages, a difference that has important implications in the discussion of the conceivability of an immortal existence.

9 Our relations with the distal stages are further complicated by 
It is true that we might be clueless about the substantive content of future practical standpoints over extremely long periods of time. But this concern is not sufficient to make us celebrate our mortality. The structure of future projection via a moving finite temporal horizon of diachronic integration is utterly familiar. By its nature, it lends itself seamlessly to a potentially indefinite extension. It has no built-in stopping point. At no moment should the agent see her life as calling for a termination simply on account of the finite extension of the horizon of temporal integration. Even if she is in the dark about what her life is going to look like beyond the horizon, she also expects to get there, more or less gradually, while always being at the center of a moving temporal horizon of identification and integration. The distal future might constantly remain unfathomable to the agent, but as she moves along, so does the horizon of the unfathomable future.

This is not to deny that one might anticipate that, at some point, one's life won't be worth living further, even if it could extend forever (or maybe because it would extend forever). But this might raise an issue for immortal life as such only if the problem were to arise for all agents necessarily and not just in particular circumstances. One might argue so only if one could show that all goods are eventually exhaustible. If so, even a life of moving horizons might eventually run into inescapable and persistent boredom, even if one were to allow for the repetition of some cycles in changing practical standpoint. ${ }^{\text {Io }}$ The issue of the exhaustibility of goods is an important one and it is worth of further investigation. But even if one were to prove that there are no inexhaustible goods, this does not yet show that we cannot coherently conceive of an immortal existence. At most, it would show that such existence would be eventually undesirable. This would, however, be as a re-

the possibility that, over the long term, we might find our survival to be threatened, even if the changes have been gradual and continuitypreserving. Altshuler (2016) interestingly argues that the existence of this threat might depend on whether we take a forward-looking or a backward-looking perspective toward the distal stages, a difference that has important implications in the discussion of the conceivability of an immortal existence.

${ }^{10}$ For some recent discussions of boredom and immortality, see Burley (2009a), Bortolotti and Nagasawa (2009), and Fischer and MitchellYellin (2OI4). 
sult of a different question than the one that we are pursuing here. What we are interested in here is only whether the temporal structure of an extended existence would by itself make an immortal life, even one with finite horizons of integration, unconceivable.

2.2. Io To sum up, the narrative view is correct in suggesting that the concept of a narrative might help describe and explain a distinctive temporal structure, one that we find both in many of our extended activities and in large portions or stages of our existence. But the narrative view is mistaken in thinking that a narrative both (a) requires closure in the form of a temporal terminus or ending, and (b) necessarily applies to each life as a whole. Without these two assumptions, however, the narrative view cannot prove that our mortality is a constitutive factor of the kinds of lives that we can coherently conceive. The narrative version of the reconciliatory strategy, therefore, cannot show that the very idea of our immortal existence is conceptually confused. As such, it fails to offer any consolation at the prospect of our eventual death.

\section{Mortality and Diachronic Agency}

\section{I A New Reconciliation?}

3. I. I Let's now consider a different version of the reconciliatory strategy, one that focuses on the connection between necessary temporal finitude and various dimensions of diachronic agency: the distinctive character of our practical predicament, the nature and structure of deliberation, and the basic cares and values that guide our conduct.

Scheffler, once again, offers a very clear and articulate statement of this strategy. First, he argues that mortality is required to make sense of the concepts that characterize what I will call our 'plight' as diachronic agents, namely, the concepts of

loss, illness, injury, harm, risk, and danger... [which] derive much of their content from our standing recognition that our lives are temporally bounded, that we are subject to death at any moment, and that we are certain 
to succumb to it in the end. In a life without death, the meaning of all these concepts would be called into question. Yet without them, it is equally unclear what would be meant by such concepts as those of health, gain, safety, security, and benefit. (Scheffler 201 3: 97)

Second, temporal finitude determines the character and structure of practical deliberation. As Scheffler writes, in trying to imagine immortal creatures,...

we are trying to imagine creatures who have little in their existence that matches our experience of tragic or even difficult choices, and nothing at all that matches our experience of decisions made against the background of the limits imposed by the ultimate scarce resource, time. But every human decision is made against that background, and so in imagining immortality we are imagining an existence in which there are, effectively, no human decisions. (Scheffler 201 3: 99)

In addition, our values are intimately related to temporal finitude. Scheffler (2013: 205) claims that mortality is a condition on our being "guided not just by our values, but by any value at all." As he further explains,

[it is unclear] what place there is for human values in such an [immortal] existence, for consider the extent to which our assignments of value are a response to the limits of time. These limits, and especially the constraints they impose upon us in the contexts of decision, force upon us the need to establish priorities, to guide our lives under a conception of which things are worth doing and caring about and choosing. Without such limits, it is at best unclear how far we would be guided by ideas of value at all. (Scheffler 2013: 99)

Finally, here is a nice summary of Scheffler's overall view:

the aspects of life that we cherish most dearly-love and labor, intimacy and achievement, creativity and humor 
and solidarity and all the rest-all have the status of values for us because of their role in our finite and bounded lives. ...[O]ur confidence in the values that make our lives worth living depends on the place of the things that we value in the lives of temporally bounded creatures like ourselves. (Scheffler 2013: IоO)

3.I.2 I will use the expression 'concepts of diachronic agency' (or CoDA) to refer to the set of all these notions that, as Scheffler convincingly argues, are at the core of our distinctive kind of existence. CoDA comprises all the concepts that characterize our plight as temporal agents, including those that characterize (i) the nature of deliberation, decision, and choices; and (ii) the character of our distinctive cares and values. The new version of the reconciliatory strategy can thus be formulated as follows: our mortality, i.e., our necessary temporal finitude, is constitutive of the conceivability and potential desirability of any existence that, like ours, is essentially and distinctively characterized in terms of the "concepts of diachronic agency' or CoDA.

3.I.3 I have no qualms about Scheffler's claim about the role of CoDA in the conceivability of our distinctive kind of temporal existence. I also find prima facie plausible that mortality might be sufficient to ground CoDA. But the reconciliatory strategy requires a stronger claim: that mortality is necessary to ground CoDA.

In the rest of this paper, pace the reconciliatory strategy, I will show that what is truly essential to CoDA is a more general form of finitude-the scarcity of opportunities for action. Although mortality is usually sufficient to generate this scarcity, I will argue that it is not necessary. In principle, we can conceive of a kind of immortal life that supports CoDA and it is thus both recognizable and potentially desirable. If so, we cannot find any consolation for our mortality at the thought that any longing for immortality is necessarily conceptually confused.

\subsection{CoDA and Vulnerabilities}

3.2. I Let's begin by noticing that there is a common feature to the elements of CoDA. More or less directly, all these concepts 
concern our vulnerability to different kinds of 'losses' or 'negative occurrences.' The first and simplest kind of loss is associated with the experience of pain. Second, there is the loss of 'disappointment,' which we experience when expectations and desires are frustrated. Third, there is what might be called the 'loss of standing' or 'status,' which is generated by betrayal, neglect, abandonment, discrimination, and the like. ${ }^{\text {I }}$

These three sorts of loss do not necessarily depend on temporal finitude. One can make sense of and be subjected to these losses independently of one's mortality. This is easy to see in the case of pain, whose occurrence might be a temporally local matter: the experience of pain at any particular moment can be utterly independent of one's states, including other pain, at other past and future moments.

3.2.2 The losses of disappointment and status, instead, might have a diachronic dimension. In particular, a disappointed attitude or a lost status is often acquired well in advance of the time of the loss. The content of the attitude or the nature of the status often depend on or concern the subject's prior history. Nonetheless, a disappointment or a deprivation of status might count as a loss at the present time regardless of the subject's future conditions. The fact that, at a later time, the attitude might come to be satisfied or the status might come to be regained does not make the present loss any less real. To this extent, these losses might be temporally local (even if one might continue to suffer them as long as the disappointment or the loss of status persists). A fortiori, whether we can experience these losses is not directly related to whether our existence is finite or infinite.

3.2.3 This is not to deny that the significance and the size of these losses might be affected by one's past or future. For instance, the experience of pain might turn into suffering in part because of the history of one's life and its narrative structures (see Dennett i 996: i6o ff). Similarly, the losses of status and standing might become much more serious on account of future harms that one is going to suffer as a result of that loss (such as the costs of frustrated assur-

${ }^{\text {II }}$ For a more general discussion of the basic vulnerabilities of agency an their connection to practical reason, see Bagnoli (2016). 
ances, the privation of benefits associated with the lost standing, etc.). But we can make sense of, and be susceptible to, the basic form of these losses independently of the future unfolding of our lives and their expected duration. Temporal finitude is not a constitutive condition of these losses.

Some of the elements of CoDA, therefore, do not seem to be related to mortality. Pace Scheffler, we do not need to conceive of our lives as temporally bounded to make sense of some versions of notions such as 'illness, injury, and harm' and of our responses to them by way of such things as 'love,' 'intimacy,' and 'solidarity.' We can easily conceive of forms of comfort and help for agents who suffer pain, disappointments, and loss of status that are understandable and meaningful regardless of the mortality of those involved.

\subsection{Scarcity of Opportunities}

3.3. I Consider now the losses associated with limited opportunities for action. As the agent's existence unfolds, she can be modeled as tracing a particular trajectory in the space of all the states she could in principle occupy. At various moments, the agent's particular trajectory is determined by the choices she makes over alternative options, that is, over possible alternative trajectories (or better, over the sets of repeatedly branching trajectories, given that in most cases she is expected to face more decision points along any of the paths she is going to choose from).

All the possible paths that the agent might take over her lifetime form a complicated web of mostly divergent and occasionally convergent trajectories in the state space. The web of trajectories constitutes the complete map of the agent's own 'garden of forking paths.' Some of the areas in the state space might be absolutely inaccessible, since no possible trajectory might reach them starting from the agent's initial position at the very outset of her existence. Other areas, instead, are only relatively inaccessible. They become unreachable only as a result of some events or choices, which put the agent on trajectories that could no long reach these areas.

Each individual trajectory is associated with the places, ob- 
jects, organisms, and people that one might encounter; with a variety of experiences, feelings, and thoughts; and distinctive failures and accomplishments. Each trajectory is also associated direct costs and benefits (including the resources that need to be expended, the effort and labor involved, etc. to move along it). The various features of any particular trajectory can be compared with those of the alternative trajectories one is choosing from. Some of the features and elements of the forfeited trajectories are going to be permanently lost, they could never again be enjoyed by the agent regardless of her future choices. The elements that are lost are the 'permanent opportunity costs' that one incurs in choosing any particular path. ${ }^{12}$ Notice that an agent might be willing to incur the opportunity costs associated with her choice, especially if none of the alternatives is appealing. An opportunity cost is not necessarily a substantive price that one is actually going to pay. But the opportunity costs represent what the agent is permanently giving up in making her choice.

For the purposes of this paper, we are not interested in how a rational agent is to go about comparing and choosing between alternative specific paths. What matters is only the basic structure of the web of branching trajectories. As we intentionally move in this space, many of our choices are among alternatives that carry permanent opportunity costs. The alternatives we choose from often have features and elements that are not fungible. Therefore, whenever we make a choice, we cannot but permanently sacrifice all of these non-fungible items.

3.3.2 Whenever some alternative paths are not fungible and they become inaccessible after the choice, we can be said to operate under 'structural scarcity of opportunities' (hereafter, unless noted otherwise, I will use 'scarcity' to refer to 'structural scarcity of opportunities'). This scarcity is structural. It is entirely a matter of

I2 There is also a different kind of opportunity cost one incurs in one's choices: the risk of the higher expenses associated with retracing one's steps if one changes one's mind and tries to take a previously forfeited path that is still accessible. But if the agent ends up retracing some of her steps, and she actually pays these expenses, she is also thereby incurring correlated permanent opportunity costs, since she is depleting scarce resources, which eventually leads her to permanently giving up some other alternatives at a future time. 
the topology of the web of trajectories, that is, of which sets of alternatives are mutually inaccessible and not fungible at any given point in the state space. But the scarcity is not a matter of either the number or the goodness of the alternatives. Structural scarcity only requires that there is at least one non-fungible and eventually inaccessible path, regardless of how good or bad this path might be in substantive terms. (Hence, there is structural scarcity even if all the alternatives that are made inaccessible by one's choice are so bad that no rational agent would ever choose them.)

This is not to deny that permanent opportunity costs might often amount to a substantial loss. Even if the agent has made the best possible choice, the paths not taken might contain valuable elements that the agent has permanently forfeited and for which there is not going to be any adequate compensation along the chosen path. How burdensome the choices are going to be depends, therefore, on the 'geography' of one's state space, That is, on the combination of (i) the topology of the web of possible paths with (ii) the substantial nature of what one is expected to experience, encounter, and achieve in each particular path.

3.3.3 The geographies of state spaces might vary greatly both in the complexity of the web of paths and in the extent to which they exhibit structural scarcity. There is something noteworthy, however, about the structural scarcity of our world. This scarcity has two distinctive properties. First, it is not limited to smaller and insignificant portions of alternative paths, which explains why alternative paths are not usually fungible. Second, choices tend to have cumulative permanent effects. As time goes by, progressively larger and larger areas of the state space become inaccessible as a result of new choices. Often one cannot retrace one's steps and the actual path one takes gets more and more entrenched into a particular area of the state space, while the rest of the state space becomes increasingly remote and eventually utterly inaccessible. As a result, one continues to incur opportunity costs even for paths that have been forfeited in the remote past. Opportunity costs might never stop accumulating, especially if, as it is common in our world, the paths continue to fork and diverge from each other, leaving little room for any future retracing or for their converging in a shared landing area at a later time. 


\subsection{Scarcity and CoDA}

3.4. I Let's get back to the discussion of CoDA-the distinctive concepts of diachronic agency. What I am going to argue in this section is that at the basis of CoDA is not temporal finitude but the structural scarcity of opportunities (henceforth, unless otherwise noted, I will drop the qualification 'structural').

It is easy to see how the scarcity provides the necessary background for deliberation, decision, and genuine choice. These activities and the related concepts make sense only in light of the constraints imposed by scarce opportunities. Not much would be left, if anything, of notions such as time-management, risk, urgency, labor, achievement, and creativity for agents whose trajectories do not have the topology distinctive of this scarcity and, therefore, never incur any permanent opportunity costs.

Scarcity of opportunities is also at the core of our plight as temporal agents. Our distinctive predicament is ultimately a matter of the pressures we face in plodding through the garden of forking paths. Simpler aspects of notions such as 'loss, illness, injury, harm, risk, and danger' (see r) might be independent of the comparison with missed or forfeited opportunities. But these notions can be fully understood only by considering how they affect agents who operate under scarcity. For what the agent loses (and what she might risk in order to avoid potential losses) is often best assessed in terms of the substantial opportunities costs that she pays when illness, injury, or harm force her to take lesser options at the time of choice.

Similar considerations can be made even for those vulnerabilities that, in their simpler form, have minimal temporal dependencies-like our vulnerability to pain, disappointment, and loss of status discussed above (see 2). This is because these losses might become much more poignant when characterized in terms of the opportunity costs that one incurs as a result of suffering them. In addition, opportunity costs are relevant to the understanding of our biological and social vulnerabilities, since biological and social harms are usually most serious when they reduce our abilities and opportunities for action. 
In turn, the ideas of solidarity, care, friendship, intimacy, and love come to their fullest expression only when they are deployed in response to the misery that we might suffer in handling scarcity and the correlated losses, harms, and sufferings. In sum, the characteristic fragilities and vulnerabilities of our condition, and the virtues and vices associated with our handling of them, are ultimately inseparable from structural scarcity.

Additionally, there is often a narrative dimension to our activities and experiences, and narrative patterns are in part comparative in nature: the kind of significance that a narrative pattern gives to the elements of the activity or experience is not based simply on how the elements cohere with each other, but also on how these elements come together by comparison to the opportunities that, for better or worse, have been forfeited in trying to bring about the unified activity or experience. ${ }^{13}$

3.4.2 Last but not least, the comparison with the paths not taken is at the root of a distinctive emotional manifestation of diachronic agency-regret. Regret is the affective response that befits our appreciation of the loss of valuable opportunities. We can regret both retrospectively (because of the forgone opportunities) and prospectively (because of the opportunities that we can anticipate sacrificing as a result of our present and future choices). In addition, we continue to incur opportunity costs throughout the unfolding of our life for the opportunities that we have forgone, and thus the regret associated with this loss can continue to increase as time goes by.

Notice that the regret induced by choosing under scarcity is not based on any negative assessment of the correctness of our choices. There can be no fault or blame attached to a choice that generates this regret. The regret in question is simply the manifestation of our appreciation that something of value has been irretrievably sacrificed, not because of our actions but because of the unfortunate structure of the world, i.e., its structural scarcity. This

${ }^{13}$ The idea of this comparison is related to what Rosati (2013: $\left.\$_{3.2}\right)$ calls the 'principle of imaginative possibilities,' according to which "an individual could not be an agent [and a practical reasoner], a being with the capacity for self-governance, unless she has the capacity to imagine otherwise" in deliberating and deciding what she will do. 
regret is thus the characteristic emotional manifestation of our plight as agents who have to make choices in a world of structurallyscarce and path-dependent opportunities. ${ }^{\mathrm{I}}$

\subsection{Scarcity and Mortality}

3.5.I How is the scarcity of opportunities related to mortality and temporal finitude? For Scheffler (2013: 99), the basic sources of CoDA are the "limits imposed by the ultimate scarce resource, time." And our necessary mortality, in turn, is what makes time scarce for us. Scheffler is correct that, given the standard topology of our state space, death makes time scarce for us and severely restricts the opportunities available to us. But this does not yet show that death and temporal finitude are indeed necessary (or even sufficient) to ground CoDA, which is what the reconciliation strategy needs to show if we are to be reconciled with our mortality.

Before proceeding further, let me clarify the important distinction between temporal finitude and temporal scarcity. Whereas finitude entails scarcity, the reverse does not hold. When some opportunities are scarce, the time to pursue these opportunities is scarce, but this is not necessarily because time itself is in general scarce. Time might be infinite, even if the interval in which we have to take certain opportunities is not. Likewise, one's individual existence might be temporally infinite even if the time to take specific opportunities is not. But the latter temporal scarcity is primarily a matter of the scarcity of opportunities, not of any temporal finitude. The reconciliatory strategy, therefore, needs to show that the grounds of CoDA lie not just in temporal scarcity but in temporal finitude, since mortality is a matter of finitude not just of scarcity. ${ }^{\text {s }}$

${ }^{\mathrm{I} 4}$ For further discussion of the role of regret, scarcity, and mortality, see Rosati (2007: 247, 255) and Rosati (2013: \$3.2). For the connection between value and regret, see Bagnoli (2000).

is Unfortunately, it is easy to slip from talk of temporal finitude to talk of temporal scarcity. This happens occasionally in Scheffler's own work. In his insightful comments on Scheffler, Kolodny (2013: 166-7) correctly points out that temporal scarcity does not require a finite existence and argues that many of the features that Scheffler links to mortality can be generated by temporal scarcity alone. I am entirely in agreement with 
3.5.2 In order to discuss how mortality and structural scarcity are related, let's first consider a scenario where the opportunities for action are structurally abundant. In this scenario, although the accessible opportunities might be limited in number, the following three conditions hold:

I. There are no opportunity costs associated with choosing one option over any other, because all the open options are utterly fungible (either because (a) these options are perfectly equivalent in terms of the experiences, encounters, and achievements that they make possible, the effort that they require, and the outcomes that they are expected to produce, or (b) the options, although they are not equivalent, are never permanently forfeited, since any option that is forfeited at a time is going to be available once again in the future);

2. There is tolerance for errors and delays;

3. The agent has sufficient ability and time to go through all the available options in any order whatsoever, even taking into account possible errors and delays.

When these conditions hold, the space of opportunities is structurally abundant. The abundance concerns the relation between the agent's choices and the available courses of action. As in the case of structural scarcity, this abundance is not a matter of how numerous or good the available options are. For instance, an agent might be in a structurally abundant situation even if she only has two open options, and both are terrible. What abundance requires is only that these two options continue to remain open to the agent no matter which one she chooses to pursue first. Conversely, the agent might have many more and much better options available to her, and yet be considered to be in a structurally scarce scenario, if her choices necessarily incur the cost of permanently forgoing some opportunities.

Kolodny on this point and much of my arguments in these sections are trying to make a similar point although in more detail than Kolodny could do in his short remarks. Let me reiterate, however, that what Kolodny says about temporal scarcity should be reformulated in terms of scarcity of opportunities, which, as I am arguing in this paper, is the ultimate source of the temporal scarcity at issue in the present discussion. 
3.5.3 Under structural abundance, one is still exercising diachronic agency but only in its minimal form. As temporal agents, we are limited in what we can immediately control and accomplish. As long as we have some distal goals, we usually need to take distinct momentary steps over a longer interval to accomplish these goals. This is the minimal temporal constraint on agency, which imposes an instrumental order over the momentary steps. Under abundance, a temporal agent only needs to make sure that, within the generous time allotment, she sooner or later takes the required steps in the proper order. But there is no urgency and no concern with lost opportunities. ${ }^{16}$

It might still seem that this temporal agent would have to worry about temporal positional goods. But under abundance, the positional character of these goods is only nominal, not substantial. Although it is true that one particular agent might get to do a certain thing or occupy a certain position earlier than other agents, that same thing and place (or a perfectly fungible one) will be available to others at later times. Hence, it does not seem that under scarcity it truly matters whether one gains any positional goods or not (although it might still matter that one acquires the goods in question, but not relative to their temporal position). Positional goods (both temporal and spatial) are genuinely important only under the relevant structural scarcity.

3.5.4 Diachronic agency under structural scarcity takes a more familiar and complex form than diachronic agency under abundance. The agent is expected to do more than just manage the momentary steps that are instrumentally required within each activity. Under scarcity, she needs to pay attention to opportunity costs, both in choosing among her options and in the implementation of the chosen alternative. She has to manage her scarce resources, including time, knowing that she is permanently forgoing some alternatives and that she is taking the risk of committing costly and possibly irremediable errors.

This is very different from the minimal exercise of diachronic agency under abundance. Because of its minimal character, agency

\footnotetext{
${ }^{16}$ For a more general discussion of the various dimensions of temporal agency, see Ferrero (20 I 2).
} 
under abundance does not ground CoDA. ${ }^{17}$ This is so even if the agent is mortal: as long as one's death is expected to come after one has enjoyed all that the structural abundance has to offer, time is not a scarce resource even if it is finite. This is because, under abundance, at some point additional time would not add any more opportunities. Once one has had sufficient time to go through all the permutations of the available options, any additional time would only allow for repetition of choices that have been made or could have been made before. ${ }^{18}$

${ }^{17}$ At most the agent might regret getting stuck in a structurally abundant but otherwise undesirable situation.

${ }^{18}$ If there is something good in the repetition of an activity already pursued in the past, the repeated activity is not strictly speaking 'exactly equivalent' to the earlier one. This is because the repeated activity carries the additional value, whatever that is, generated by the repetition. If repetition brings about any additional value, then time would indeed be scarce if there is not enough time to allow for the repetition-because this repetition would effectively count as a novel opportunity one would be deprived of if time is cut short. Thus, genuine structural abundance requires that, given the limited set $S$ of the opportunities that it leaves open, there be enough time to go through as many rounds of all the elements of $S$ as there continue to be valuable repetitions. An interesting question arises at this point: is the repetition of something valuable always valuable, even ad infinitum? One way in which repetition might always be valuable is that it allows for one to continue existing. But if so, the value is ultimately in the sheer continuation of one's existence, regardless of the independent value of what gets repeated. For a discussion of the possible desirability and value of simply being, see Nagel (1970) and Rosati (2013: \$3.I). Others might instead argue that there is always additional substantive value due to the distinctive character of the repeated activity itself. If so, there is always a pressure in favor of endless repetitions. But a worry arises in such scenarios: could an infinite existence in the mode of valuable repetition continue to support CoDA? This existence is one of structural abundance except that opportunities for repetition would become scarce if one were to die. However, there is no loss associated with any of the choices that one is making while going through the elements of the abundant set $S$. If so, it seems that we are facing a very different kind of opportunity costs than the one associated with the structural scarcity induced by the branching of mutually inaccessible paths. What we lose in this scenario is only the opportunity for some more encores, which is not exactly the kind of loss associated with the elements of CoDA that we have been discussing thus far. This is a very interesting issue but one that, unfortunately, needs to be taken 
3.5.5 The temporal finitude of one's life gives rise to CoDA only in so far as it contributes to generating structural scarcity. This is how temporal finitude contributes to scarcity in our lives, but this finitude is neither a sufficient nor a necessary condition for structural scarcity. As long as there is structural scarcity of opportunities, there are the grounds for a recognizable existence, for an existence that exhibits all of CoDA, regardless of its expected duration.

\subsection{Endless Scarcity and Immortality}

3.6. I Given that CoDA are grounded in structural scarcity of opportunities but not in temporal finitude, I surmise that we might conceive of agents who can have an immortal life in a universe of perpetual structural scarcity. This is a universe in which the possible paths continue to branch out and, when not taken, become perpetually inaccessible. As an agent moves in this space, she can forever continue to incur opportunity costs-she irremediably loses some possible experiences, encounters, and achievements, and she suffers from the permanent separation from objects, organisms, places, and persons. But in this universe, the agent's losses do not require her to be mortal. Nor does the permanent separation from objects, organisms, places, and persons require that any of these, especially other persons, be transient. All that it takes for these losses to be real is that there are opportunities and entities that become permanently inaccessible as she moves in the unending garden of continuously and permanently forking paths.

A web of opportunities with this infinite structural scarcity is in principle conceivable. In a universe that supports this scarcity, therefore, there could be immortal agents whose existence supports CoDA. Hence I conjecture that there is a conceptually coherent kind of immortal existence that we can in principle recognize and potentially desire. As long as this conjecture stands, one can no longer claim that mortality is a necessary condition of

up on another occasion. For the role that some repeatable activities and experiences might play in the desirability of immortality, see Fischer's important proposal in Fischer (1994), Fischer (2013), and Fischer and Mitchell-Yellin (2014). 
CoDA. And if so, the reconciliatory strategy is in trouble. It can no longer claim that that we must reconcile with mortal existence as the only conceptually coherent one. ${ }^{19}$

3.6.2 It is notoriously difficult to offer a positive proof of a conjecture about conceivability, so I won't attempt one here. But I will try to garner some indirect support for it by rejecting the most serious objection to it-the claim that an immortal agent would necessarily and permanently enter into a condition of structural abundance.

There are two ways to argue for the necessity of structural abundance over an infinite existence. First, one might claim that an infinitely long existence is ipso facto an existence of maximal substantial abundance. This is an existence where all possible permutations of all possible opportunities will sooner or later occur so that no opportunity for action is ever really permanently inaccessible. Some opportunities might be temporarily blocked, so that the immortal agent might still need to exercise minimal diachronic agency in handling a temporarily limited range of options. But the opportunity costs are only local and transitory. They are completely reversible at a later time when one can once again choose to follow paths that were previously passed up.

3.6.3 Second, one might argue for an 'end of history' scenario: an infinitely long existence necessarily takes the agent into a permanently restricted structural abundance. That is, a scenario in which there is only a limited set of open options but such that all of these options are permanently available in the mode of structural abundance (see 2).

This 'end of history' strategy seems harder to pursue. One

I9 Rosati, who correctly insists on the importance of the scarcity of opportunities for the understanding of the distinctive features of our existence and agency, at some point calls this scarcity 'a kind of mortality that arises repeatedly within a life,' Rosati (2007: 244, my emphasis). This is a bit disingenuous and should not be read as a concession to the reconciliatory view. It is just a way for Rosati to highlight that there is a pervasive finitude in our existence, one that we might think akin to genuine mortality but that, as I am arguing here, should not really be confused with it. 
would have to show that under all possible circumstances, any endless existence would necessarily lead into some kinds of restricted structural abundance. But why should we think that an infinite temporal extension necessarily brings about the shrinking of available opportunities under all possible conditions? If anything, the thought of infinite existence seems to suggest the opposite: the maximal availability of opportunities of the first scenario-that of maximal substantial abundance. The best case for restricted structural abundance is to suggest, with Smuts (2OI I), that immortal agents would have a fixed set of limited abilities and, therefore, would eventually run out of things they could accomplish. But why should we think that immortality entails that agents have fixed abilities? And even if it did entail this, why should we think that there are no goals or projects that could be pursued indefinitely by agents with fixed abilities? It does not seem to me that one could establish either of these two assumptions simply on the basis of the notion of an immortal existence.

3.6.4 The first strategy-that of maximal substantial abundanceappears more promising. In this case, it is clearer how this abundance is supposed to ensue from immortality. The thought is that for any immortal agent, over the infinite amount of time of her existence, every possible combination of opportunities for action would, sooner or later, become available (see Smuts 20 I I: I43, and May 2009). Those who find this picture of immortality plausible insist on its unrecognizability. They describe it as 'motivationally devastating' and 'shapeless,' as lacking any of the distinctively human urgencies, losses, and regrets, and as devoid of meaning and significance (see in particular May 2009: 68, Smuts 2008, Smuts 20 I I, and 'early' Nussbaum 1994).

These philosophers are correct in pointing out the devastating effects of maximal abundance. These effects are exactly what we should expect given that maximal abundance leaves no room for CoDA..$^{20}$ But to reject my conjecture that immortality might be compatible with perpetual scarcity, one needs to establish that

\footnotetext{
${ }^{20}$ Notice that CoDA would still get a foothold in the 'end of history' scenario. This kind of immortal life, therefore, could be recognizable and potentially desirable at least up until the time when one transitions into the condition of permanent structural abundance.
} 
immortality necessarily gives rise to maximal abundance. Unfortunately, no such argument is offered by those who equate immortality with abundance. In their writings, they simply assume what Borges (I964: I I4) tells us about the immortals, that they know "that in an infinite period of time, all things happen to all men." ${ }^{21}$

3.6.5 Those who equate immortality with abundance assume what might be called a 'combinatorial' view of infinite existence. In so doing, they ignore at least two other basic ways in which infinite time might affect the circumstances of action. First, the 'cyclical' model (as exemplified by eternal recurrence) in which the same set of circumstances (and, possibly, of the corresponding actions) repeats itself over and over ad infinitum. Second, the 'directional' model that underlies my conjecture, where infinite time continues to allow for some persistent scarcity of opportunities.

I do not think that the notion of an infinite time by itself rules out any of these three models of immortal life. This is all that I need to show for my conjecture to succeed in undermining the reconciliatory account. Although the combinatorial model (and possibly the cyclical one as well) might be incompatible with a recognizable existence that supports CoDA, the directional model is compatible with scarcity and thus with CoDA. Hence, as long as the directional model is not ruled out by the very idea of infinite time, my objection to the reconciliatory account still stands.

\subsection{Contingent Immortality}

3.7.I Up to this point, my discussion has been framed according to the standard interpretation of both mortality and immortality as necessary conditions, that is, mortal beings necessarily die, and immortal ones necessarily live forever. ${ }^{22}$ But there is a third notion that is rarely discussed, that of contingent immortality. For a

${ }^{21}$ See also the description of the Homeric gods in Nussbaum (1994), "who can easily do anything they want any time they want," Nussbaum (2013: 36).

${ }^{22}$ Or more generally, immortality is interpreted as guaranteed: either one necessarily lives forever or one is guaranteed to continue to live as long as one chooses to do so, as it happens in the case of Elina Makropulos in Williams (1973). 
contingently immortal agent death is not necessary. Such an agent might end up living forever provided she continues to succeed at overcoming the potentially lethal threats that she might encounter throughout her existence. ${ }^{23}$

3.7.2 Does the notion of contingent immortality make a difference to my argument against the reconciliatory strategy? I am going to argue that it doesn't. If immortality is not guaranteed, an agent can be said to be 'potentially immortal.' Such an agent could become actually immortal only by a combination of skill and luck. She needs to be lucky with respect to the potentially fatal events over which she has no control. She needs skill, instead, to handle the threats over which she has some control. To the extent that her continuous survival depends on her skills, her immortality would be an achievement. But an achievement that she might never reach once and for all, if potentially lethal threats continue to arise for ever and ever.

A potentially immortal agent might incur some opportunity costs that are very similar to those of a necessarily mortal agent. Both kinds of agent face the dangers to lose their lives. The opportunity costs for the potentially immortal agent might actually be much higher since she has much more to lose in dying. She would lose a potentially infinitely long life, rather than just a longer one. Because of the danger of death and the need to take risks with respect to it, there is a central dimension of the plight of potentially immortal agents that is utterly missed by agents whose immortality is guaranteed, even when the latter ones operate under persistent structural scarcity.

3.7.3 It might be argued that contingent immortality shows that there is more to CoDA than just structural scarcity. In particular, structural scarcity cannot account by itself for the distinctive achievement of skillfully avoiding or overcoming potentially lethal threats. Agency-it might be plausibly argued-can be fully understood only in connection with the notion of a life that requires 'survival' in the face of the ultimate danger of death. That is, we

${ }^{23}$ A version of contingent immortality is introduced by Steele (1976) and gets some rather limited discussion in Burley (2009b), Scheffler (2O I3), and Fischer (2OI3). 
can make sense of agency only in connection with mortality in the sense of the constant liability to (rather than the ineluctability of) death. Or, in other words, we can make sense of agency only together with the power to keep oneself alive, i.e., the power of self-maintenance against the threat of annihilation.

This power of self-maintenance need not be interpreted only in biological terms. The survival of rational agency might require a form of psychological self-maintenance in response to a fundamental ontological vulnerability of the rational and psychological unity of an agent. Following Korsgaard (2009), one might plausibly argue that a rational agent is the product of a process of self-constitution in response to potentially disruptive psychological forces (which, in principle, might operate independently of the vulnerabilities generated by our biological and physical nature). If so, the temporal existence of an agent might require not just the CoDA generated by structural scarcity but also those related to her potential mortality, to her constant facing and struggling with the danger of death.

3.7.4 How would the prospect of reconciliation look like if we consider this kind of potential mortality? Let's first distinguish between two kinds of liability to death: (a) liability to fatal events outside of our control, and (b) liability to irremediable failures of self-constitution.

In being liable to death by uncontrollable events, we are distinctively fragile: our survival is ultimately hostage to fortune. This fragility shapes in substantive ways our actual lives. It would also shape in similar ways the lives of potentially immortal agents. This liability, however, does not seem to be necessary for the conceivability of a temporal existence as a diachronic agent. In this way, it is unlike both the scarcity of opportunities and the liability to failures of self-constitution. Thus, I contend, we might have no reason to reconcile with this mortality as the liability to uncontrollable fatal events. We might justifiably lament this fragility, even while we acknowledge that it is an inescapable feature of our lives.

3.7.5 By contrast, it should not be difficult to reconcile ourselves with the liability to irremediable failures of self-constitution pro- 
vided that these failures are ultimately due to catastrophic errors in the exercise of our own rational powers. We might desire that the job of rational survival be easier. But we cannot but accept the liability to these catastrophic errors if this liability is indeed shown to be a necessary precondition of the very possibility of our being under the guidance of the demands of rationality and, thereby, of the very possibility of our continuing to exist as rational agents. ${ }^{24}$ In other words, we should accept all the toil and anxious striving of rational self-constitution as long as it is the necessary counterpart of our existence, and as long as the blame for any fatal failure would ultimately rest only on ourselves as we try to exercise our rational powers.

This reconciliation with liability to fatal failures of rational self-constitution does not help, however, to reconcile with mortality as the necessity of death. Being liable to failures of selfconstitution, by itself, does not rule out that our rational powers, when properly exercised, might secure a continuous and potentially indefinite survival under the proper conditions. What might be constitutive of diachronic agency is only our subjection to the constant danger of death by a catastrophic failure of selfconstitution, not the inevitability of our eventual demise by an actual failure.

\subsection{Cosmic Regret}

3.8. I The conclusion of the previous section shows that, even if we add the liability to failures of self-constitution to structural scarcity, we can still conceive of an immortal existence that grounds CoDA. Let me stress once again that I am only offering a conjecture about the conceivability of a certain kind of immortal existence. I am not arguing that in our actual world time is both infinite and supportive of perpetual structural scarcity.

I am only concerned with conceivability because this is all that is required to assess the fate of the reconciliatory strategy. To be reconciled with our necessary mortality, we need an antidote

${ }^{24}$ For a general discussion of the relation between liability to error and rational guidance, see Lavin (2004). 
against what might be called 'cosmic regret.' This is the regret we might experience when realizing that, just in our capacity as temporal agents, we could conceive of a mode of existence that retains the core features of diachronic agency without necessitating death. When so, we might feel disappointment and sorrow at the thought that we live in a world that does not allow us to have a recognizable (and potentially desirable and meaningful) immortal life. Even if we acknowledge that many attractive features of the human condition would be lost in such a universe, this does not prevent us from seeing something appealing in this immortal existence and to find lamentable that we are deprived of it.

3.8.2 This is regret is different from both agent-regret-which concerns the effects of our faulty or blameworthy actions-and choiceregret-which concerns the opportunities we missed or forfeited as a result of our choices, whether they were correct or not. Cosmic regret, instead, reflects both (i) our understanding of the basic nature of diachronic agency and temporal existence and (ii) our acknowledgement that the universe could have turned out differently; that it could have allowed for us to be potentially immortal while retaining both our capacity for diachronic agency and the grounds for CoDA.

Is the avoidance of cosmic regret too high a desideratum to impose on any successful reconciliation with mortality? I do not think so. In attempting the reconciliation, the stakes are very high. We are trying to figure out what to make of the most basic aspects of our existence. This is why, in these speculations, the only thing that we should keep fixed is just our condition as self-conscious subjects with the power of diachronic agency. The immortal life that we might be able to recognize (and possibly long for) might turn out to be possible only in a universe that, except for its support of the fundamental features of diachronic agency, is very remote from our own.

3.8.3 Notice that I am not denying that there is much more to our existence than just our condition as diachronic agents. We can see ourselves under thicker conceptions, including the conception as human beings, with a distinctive physical and biological nature. There are properties essential to us under these other conceptions that might make us necessarily mortal. There could be things that 
we cherish and value that that go beyond the basic elements of $\mathrm{CoDA}$ and that might necessarily depend on properties, such as our distinctive vulnerabilities as biological organisms, that make us mortal. Hence, when we look at ourselves under these thicker conceptions, we might indeed be able to find some consolation at the prospect of our necessary death. But this does not necessarily tell against cosmic regret for the immortality that is not granted to us. Insofar as we can make at least a notional distinction between our different conceptions, the one as diachronic agents and the one as human beings, say, we can still coherently project our existence in the mode of diachronic agents into an endless future.

Hence, we can at the same time both embrace and regret our necessary mortality without inconsistency. There are different dimensions and guises under which we can assess our life and the prospect of death. And in this exercise, we can compare our life to different sorts of alternative existences. Ultimately, we can harbor contrasting attitudes toward our mortality because there is more than one legitimate question that we might ask about the alleged badness of our death (see Velleman 20I 2). Hence, we should not be surprised if our stance toward mortality, although not inconsistent, is often rife with ambiguity.

3.8.4 In this paper, I am concerned with our response to mortality in the most general form, as something that might affect us under the broadest conception of ourselves as diachronic agents. It is with respect to this conception of ourselves that, I have claimed, we can feel cosmic regret for a kind of immortality that has not been granted to us.

Notice that this regret is cosmic in object, not necessarily in intensity. The very remoteness of the universe in which we might have such immortal existence might prevent us from falling into a devastating despair. In addition, in mourning this missed immortality, we must realize that the grounds of what we might find appealing about it are already available in our actual and mortal human form: the structural scarcity and the liability to failures of self-constitution. The basic structure of any conceivable immortal existence is the same as that of our actual existence, except that, for better or for worse, the immortal existence is supposed to carry this structure for a lot longer. 
3.8.5 Is the longer and indefinite duration preferable? Thus far I have always talked about the 'potential' desirability of immortality. The conceptual recognizability of immortality-cum-scarcity does not entail either the absolute or relative attractiveness of this immortality (under any of the possible dimensions under which lives might be attractive, including, as Rosati (2OI 3: \$2.2) correctly remarks, their being meaningful, morally valuable, aesthetically pleasing, or personally good).

There are two reasons why recognizability does not entail desirability. First, a persistent structural scarcity does not guarantee that the scarce opportunities are at all appealing. It is possible that all available options are most undesirable and that the opportunity costs that one incurs are not only irremediable but also a continuous and never ending source of suffering. ${ }^{25}$ Whether immortality-cum-scarcity is desirable ultimately depends on the actual geography of the state space, and the web of opportunities that it affords to any particular agent based on her specific location in that space. There is no guarantee that any given agent is always going to find at least one open path that will make her endless journey along it choiceworthy.

3.8.6 A second reason to doubt that recognizability entails desirability is the worry that, over the long run, all agents might end up with no desirable options, regardless of their starting points and subsequent choices. The concern is that there might be no genuinely inexhaustible goods. Even if structurally scarcity is perennial, so that the preconditions for the genuine pursuit of goods might never go away, the goods themselves might at some point necessarily run out.

In the discussions of immortality, it is often remarked that inexhaustible goods might be offered by such things as friendship, artistic creativity, or aesthetic experience (and possibly the contemplation of truth, goodness, beauty, or God-at least as long as this contemplation is supposed to take place over time rather than in timeless eternity). But I think we should be cautious about accepting claims of this kind if they are just based on projections into the infinite future of intuitions about goods that we have only

${ }^{25}$ For a similar worry, see Kolodny (201 3: 167). 
experienced under finite conditions. There might be principled reasons to think that these goods are indeed inexhaustible, but we need an argument to this effect.

My conjecture about the conceivability of an immortal existence also relies on a projection into the infinite time but I feel more confident about it than any claim about the goods. The considerations that I have advanced here are only based on structural considerations about the nature of diachronic agency and the possible topology of the state space and the agent's trajectories in it. This conjecture still leaves open the issue whether such a structure might be filled with inexhaustible substantive goods, a question that would have to be taken up on another occasion. ${ }^{26}$

\section{CONCLUSION}

\section{I The Failed Promise of Reconciliation}

4. I. I The reconciliatory strategy promised to offer the ultimate consolation for our mortality. It was supposed to show that we should embrace our necessary temporal finitude as constitutive of what is distinctive of our value-laden temporal agency and existence, and thereby give up the conceptually confused wish for immortal existence. Unfortunately, this strategy fails. This is not to deny that it has some merits. The strategy offers the best possible schema for ultimate consolation. It is also correct in the substantive characterization of the distinctive features of our existence. Finally, it is correct in claiming that our finitude is constitutive of these features. But there are several dimensions to our finitude and the strategy ends up focusing on the wrong one-the necessary temporal finitude of our existence. ${ }^{27}$

${ }^{26}$ There is an additional issue that has to be left for another occasion: Could it be that what is conceptually confused is not the idea of an immortal life but that of its desirability? This is an intriguing suggestion raised by Altshuler (2016). Unfortunately, I have become acquainted with Altshuler's proposal too late to properly discuss it in this paper.

${ }^{27}$ In fairness to the proponents of the reconciliatory strategy, I want to acknowledge that they often start by mentioning the more general finitude and by suggesting that this finitude is related to the distinctive 
As I have shown in this paper, there are several ways in which we are finite and bounded: our existence can be temporally finite, the temporal horizon of identification and integration can be limited, goods might be exhaustible, we might be liable to potentially fatal dangers and catastrophic failures of self-constitution, and we might be acting under structural scarcity of opportunities. I have argued that only structural scarcity (possibly combined with the liability to failures of self-constitution) is constitutive of the fundamental and distinctive features of our temporal existence as diachronic agents.

I conjectured that there might be a world that could support the existence of immortal agents who grapple with a persistent structural scarcity and thus have a life that might ground the basic features of what we recognize, cherish, and value in our own mortal existence. As long as this conjecture stands, the reconciliatory strategy cannot deliver on its promise: we cannot console ourselves for our mortality as being necessary to ground any conceivable and desirable existence.

4. I.2 Unlike many common pictures of immortality, the one I offer here is not a conceptually confused product of naïve imagination. Rather, it offers a genuine 'intimation of immortality.' Not an intimation of actual immortality but of the kind of endless existence that we, in our capacity as diachronic agents, could lead, even if only in a very remote universe and at the cost of losing many distinctive aspects of buman existence. ${ }^{28}$

This immortal existence is not only conceivable but also potentially appealing. And this is why we might regret not being

features of our existence. But then these discussions immediately turn their focus exclusively on mortality as temporal finitude. In so doing they fail to distinguish the contributions of the other dimensions of finitude and, more damagingly, they fail to realize that the reconciliatory strategy might thereby fail to establish its conclusion.

${ }^{28}$ In talking about 'intimations of immortality' I am echoing the conclusion reached by Rosati (2O I 3). Like her, I am claiming that the longing for a potentially indefinitely long existence is not conceptually confused but it actually expresses a central aspect of our agency. Where we differ is on the dimension of agency that gets so expressed. I focus on diachronicity, while she focuses on autonomy and self-governance. 
immortal. This is not to claim that we would necessarily prefer to be immortal. The goods promised by an endless existence might not be unalloyed, which would explain the deep ambivalence we often feel toward our mortality.

I suspect that, if we were to face an actual choice between a mortal and an immortal life, we would not be unreasonable in opting for the finite one. But this choice appears dilemmatic. The two options are likely to be incomparable; no matter which one we were to decide on, we could still justifiably regret the permanent loss of the goods offered only by the other kind of life. But this loss is not something we can presently deplore. Given our necessary mortality, we are deprived of the very possibility to face this dilemma.

Sometimes we are grateful to be spared dilemmatic situations. But in this case, it is lamentable that we are not offered the opportunity to make up our mind. Even if we were to settle for a finite life, we can still acknowledge that there is some incomparable value in an endless existence, a value that would be permanently lost to us in choosing to die. Hence, what we might ultimately mourn about our necessary mortality is not our eventual death but the impossibility to regret the loss of the value that we would give up in rejecting immortality.

\section{References}

Altshuler, R. (2016). Immortality, Identity, and Desirability. In Cholbi, M., editor, Immortality and the Philosophy of Death, pages I9I-203. Rowman \& Littlefield Publishers.

Bagnoli, C. (2000). Value in The Guise of Regret. Philosophical Explorations, 3: 165-1 87.

Bagnoli, C. (2016). Vulnerability and the Incompleteness of Practical Reason. In Strahele, C., editor, Vulnerability in Context, chapter 4. Routledge.

Borges, J. L. (1964). The Immortal. In Donald A. Yates, J. E. I., editor, Labyrinths: Selected Stories Other Writings. New Directions, New York.

Bortolotti, L. and Nagasawa, Y. (2009). Immortality without Boredom. Ratio, 22(3):26 I277.

Brooks, P. (2012). Reading for the Plot: Design and Intention in Narrative. Alfred A. Knopf.

Burley, M. (2009a). Immortality and Boredom: A Response to Wisnewski. International Journal for Philosophy of Religion, 65(2):77-85. 
Burley, M. (2009b). Immortality and Meaning: Reflections on the Makropulos Debate. Philosophy, 84(4):529-547.

Dennett, D. C. (1996). Kinds of Things. Harper Collins.

Ferrero, L. (2009). What Good is a Diachronic Will? Philosophical Studies, I 44(3):403430.

Ferrero, L. (201 2). Diachronic Constraints of Practical Rationality. Philosophical Issues, 22(I): I 44-I 64 .

Fischer, J. M. (1 994). Why Immortality is Not So Bad. International Journal of Philosophical Studies, 2(2):257-270.

Fischer, J. M. (2005). Free Will, Death, and Immortality: The Role of Narrative. Philosophical Papers, 34(3):379-403.

Fischer, J. M. (20I 3). Immortality. In Bradley, B., Feldman, F., and Johansson, J., editors, The Oxford Handbook of Philosophy of Death. Oxford University Press.

Fischer, J. M. and Mitchell-Yellin, B. (2OI4). Immortality and Boredom. Journal of Ethics, I $8(4): 353-372$.

Helm, P. (2OI4). Eternity. In Zalta, E. N., editor, The Stanford Encyclopedia of Philosophy. Spring 2014 edition.

Ismael, J. T. (2006). The Ethical Importance of Death. In Tandy, C., editor, Death and Anti-Death: Twenty Years After De Beauvoir, Thirty Years After Heidegger, volume 4, pages I 8 I-I 98. Ria University Press.

Kermode, F. (2000). The Sense of an Ending: Studies in the Theory of Fiction. Oxford University Press, USA.

Kolodny, N. (2013). That I Should Die and Others Live. In Scheffler, S. and Kolodny, N., editors, Death and the Afterlife. Oxford University Press.

Korsgaard, C. (2009). Self-Constitution: Agency, Identity, and Integrity. Oxford University Press.

Lavin, D. (2004). Practical Reason and the Possibility of Error. Ethics, I I 4:424-457.

Malpas, J. E. (1 998). Death and the Unity of a Life. In Malpas, J. E. and Solomon, R. C., editors, Death and Philosophy, pages I 20- I 34. Routledge.

May, T. (2009). Death. Acumen, Durham.

Nagel, T. (1 970). Death. Noûs, 4(I):73-80.

Nussbaum, M. C. (1989). Mortal Immortals: Lucretius on Death and the Voice of Nature. Philosophy and Phenomenological Research, 50(2):303-35 I.

Nussbaum, M. C. (1 994). The Therapy of Desire. Princeton University Press.

Nussbaum, M. C. (1999). Reply to Papers in Symposium on Nussbaum, The Therapy of Desire. Philosophy and Phenomenological Research, 59(3):8 I I-8 I 9.

Nussbaum, M. C. (2013). The Damage of Death: Incomplete Arguments and False Consolations. In Taylor, J. S., editor, The Metaphysics and Ethics of Death, pages 25-43. Oxford University Press.

Rosati, C. S. (2007). Mortality, Agency, and Regret. Poznan Studies in the Philosophy of the Sciences and the Humanities, 94(I):23 I-259.

Rosati, C. S. (2OI3). The Makropulos Case Revisited: Reflections on Immortality and Agency. In Bradley, B., Feldman, F., and Johansson, J., editors, The Oxford Handbook of Philosophy of Death, pages 355-390. Oxford University Press.

Scheffler, S. (2013). Death and the Afterlife. Oxford University Press.

Smuts, A. (2008). Wings of Desire: Reflections on the Tedium of Immortality. Film and Philosophy, I3(I): 1 37-15 I. 
Smuts, A. (20 I I). Immortality and Significance. Philosophy and Literature, 35 (I): I 34- I 49. Steele, H. (1 976). Could Body-Bound Immortality be Liveable? Mind, 85(339):424-427. Strawson, G. (2004). Against Narrativity. Ratio, I7(4):428-452.

Tolstoy, L. N. (1 884). A Confession and Other Religious Writings. Viking Penguin, New York.

Velleman, J. D. (201 2). Comments on John Martin Fischer's Our Stories. Philosophical Studies, I $58(3): 5$ I $5-52 \mathrm{I}$.

Williams, B. (1973). The Makropulos Case: Reflections on the Tedium of Immortality. In Problems of the Self, pages 82-1 oo. Cambridge University Press.

Williams, B. (2009). Life as Narrative. European Journal of Philosophy, I7(2):305-3 I 4. 